\title{
Incorporating Molecular Scale Structure into the van der Waals Theory of the Liquid-Vapor Interface ${ }^{\dagger}$
}

\author{
Kirill Katsov \\ Department of Physics, University of Washington, P.O. Box 351560, Seattle, Washington 98195-1560
}

\author{
John D. Weeks* \\ Institute for Physical Science and Technology, and Department of Chemistry and Biochemistry, \\ University of Maryland, College Park, Maryland 20742
}

Received: April 11, 2002; In Final Form: June 8, 2002

\begin{abstract}
We have developed a new and general theory of nonuniform fluids that naturally incorporates molecular scale information into the classical van der Waals theory of slowly varying interfaces. Here the theory is applied to the liquid-vapor interface of a Lennard-Jones fluid. The method combines a molecular field treatment of the effects of unbalanced attractive forces with a locally optimal use of linear response theory to approximate fluid structure by that of the associated (hard sphere like) reference fluid. Our approach avoids many of the conceptual problems that arise in the classical theory and shows why capillary wave effects are not included in the theory. The general theory and a simplified version gives results for the interface profile and surface tension for states with different temperatures and potential energy cutoffs that compare very favorably with simulation data.
\end{abstract}

\section{Introduction}

This paper applies our general theory of nonuniform fluids, described in several earlier publications, ${ }^{1-5}$ to the liquid-vapor interface of the simple Lennard-Jones fluid. Our approach here can be viewed as a generalization of the classical van der Waals (VDW) theory for the density profile of the liquid-vapor interface ${ }^{6,7}$ that (a) incorporates accurate thermodynamic data for the uniform fluid and (b) corrects the usual assumption that the interface profile is slowly varying. The new theory takes into account nonlocal molecular scale density correlations in a very natural way and can be applied to a wide variety of problems where the classical theory would fail. This perspective also provides a new and physically suggestive interpretation of the classical VDW theory for even a slowly varying liquidvapor interface that removes many of the conceptual problems and ambiguities that arise in standard descriptions. ${ }^{7}$ Thus it seems appropriate to refer to it as a molecular scale van der Waals (MVDW) theory. ${ }^{5}$

Since many aspects of the MVDW theory have been presented in some detail in previous work, here we will just outline the main features and focus on the new results we find for the structure and thermodynamics of the liquid-vapor interface. In this specific application, where the interface is often slowly varying, correction (a) plays the most important role and (b) is relatively less important, though conceptually (b) represents the most important advance and permits much more general application of the theory. In particular we will show how our theory, which first determines the structure of the liquid-vapor (LV) interface, can be used to calculate thermodynamic properties such as the surface tension. These results will be compared to data from computer simulations ${ }^{8-11}$ and to

† Part of the special issue "John C. Tully Festschrift."

* Corresponding author. a simplified version of the MVDW theory that includes only correction (a). We also discuss the role of capillary wave fluctuations, ${ }^{7,12,13}$ which should be taken into account when comparing theory and experiment.

\section{Molecular Field Approximation}

An essential ingredient in the MVDW theory and in our interpretation of the classical VDW theory is the introduction of an effective single-particle potential or "molecular field" to describe the locally averaged effects of the unbalanced attractive forces in the nonuniform LJ fluid. ${ }^{14}$ Since the attractive interactions are relatively slowly varying, such an averaged treatment seems physically reasonable. To that end, the LJ pair potential $w(r) \equiv u_{0}(r)+u_{1}(r)$ is separated into rapidly and slowly varying parts associated with the intermolecular forces so that all the harshly repulsive forces arise from $u_{0}$ and all the attractive forces from $u_{1}$. We describe the LV interface using a grand ensemble with a fixed chemical potential $\mu^{\prime}$ and temperature $k_{\mathrm{B}} T \equiv \beta^{-1}$ giving two phase coexistence with bulk liquid and vapor densities $\rho^{\prime}$ and $\rho^{v}$, respectively, in an external field $\phi(\mathbf{r})=0$.

The theory then approximates the structure of the nonuniform LJ system by that of a simpler nonuniform "reference" or "mimic" system at the same temperature but with a chemical potential $\mu_{0}^{\prime}$ and purely repulsive pair interactions $u_{0}(r)$. These give the same repulsive intermolecular forces as in the LJ fluid and are well approximated for most purposes by hard sphere interactions. The nonuniformity in the reference system is induced by an appropriately chosen effective reference field (ERF) $\phi_{\mathrm{R}}(\mathbf{r})$ that is supposed to take into account the locally averaged effects of the attractive interactions in the LJ fluid.

How should $\phi_{\mathrm{R}}(\mathbf{r})$ be chosen? Since we want the reference fluid structure to approximate that of the full fluid to the extent possible, it seems reasonable to determine $\phi_{\mathrm{R}}(\mathbf{r})$ formally by 
the requirement that the local (singlet) densities at every point $\mathbf{r}$ in the two fluids are equal: ${ }^{15}$

$$
\rho_{0}\left(\mathbf{r} ;\left[\phi_{\mathrm{R}}\right], \mu_{0}\right)=\rho\left(\mathbf{r} ;[\phi], \mu^{\gamma}\right)
$$

Of course this density is not known in advance, so in practice we will make approximate choices for $\phi_{R}$ motivated by mean or molecular field ideas. Here the subscript 0 denotes the reference fluid, the absence of a subscript the LJ fluid, and the notation $[\phi]$ indicates that the correlation functions are functionals of the external field $\phi$ (which in the present case is zero in the LJ system and $\phi_{\mathrm{R}}$ in the reference system). Unless we want to emphasize this point, we will suppress this functional dependence, e.g., writing eq 1 as $\rho_{0}(\mathbf{r})=\rho(\mathbf{r})$.

\section{Classical VDW Interface Equation}

As discussed in detail in ref 5, we can derive the classical VDW interface equation from this starting point by making two additional approximations. We briefly discuss this interpretation of the classical theory and then describe how our new MVDW theory improves on both approximations.

A. Simple Molecular Field Approximation. First, the classical VDW theory uses the simple molecular field (MF) approximation for the ERF $\phi_{\mathrm{R}}$ :

$$
\phi_{\mathrm{R}}\left(\mathbf{r}_{1}\right)=\phi\left(\mathbf{r}_{1}\right)+\int \mathrm{d} \mathbf{r}_{2} \rho_{0}\left(\mathbf{r}_{2} ;\left[\phi_{\mathrm{R}}\right], \mu_{0}\right) u_{1}\left(r_{12}\right)+2 a \rho^{\prime}
$$

where

$$
a \equiv-\frac{1}{2} \int \mathrm{d} \mathbf{r}_{2} u_{1}\left(r_{12}\right)
$$

corresponds to the attractive interaction parameter $a$ in the uniform fluid VDW equation, as discussed below. This is just a transcription of the usual molecular field equation for the Ising model to a continuum fluid with attractive interactions $u_{1}(r)$ and can be derived in a number of different ways. ${ }^{16,17}$ The connection to the unbalanced attractive forces is perhaps most clearly seen in the derivation in refs 1 and 3, which starts from a formally exact description of the force balance in a nonuniform fluid and arrives at eq 2 by a series of physically motivated approximations.

For the LV interface we have $\phi(\mathbf{r})=0$, but it is convenient in what follows to keep a general $\phi$, which we will then set to zero. In that case we will also choose $\mu_{0}^{\prime}$ so that the density $\rho_{0}^{\prime}$ of the uniform reference fluid with $\phi_{\mathrm{R}}=0$ equals $\rho$. With this choice the ERF $\phi_{R}$ vanishes on the liquid side far from the interface where the density becomes equal to $\rho$.

Another special case of eq 2 arises when $\phi$ is a constant. Since a constant field in the grand ensemble is equivalent to a shift of the chemical potential, eq 2 then relates the chemical potentials in the uniform LJ and reference fluids. ${ }^{3}$ Equation 2 thus yields the familiar uniform fluid VDW result ${ }^{7}$

$$
\mu(\rho)=\mu_{0}(\rho)-2 \rho a
$$

where $\mu(\rho)$ and $\mu_{0}(\rho)$ denote the chemical potential as a function of density $\rho$ for the uniform LJ fluid and the reference fluid, respectively. In the classical theory $\mu(\rho)$ is defined for all $\rho$ in terms of known reference system quantities by the right side of this equation. Since the uniform reference fluid is well defined for all densities below freezing, no problems arise from densities in the two phase region of the LJ fluid. The MVDW theory will use a slightly different expression for $\phi_{R}$ in eq 11 below that gives a more accurate description of the thermodynamics of the uniform LJ fluid.

In general, to calculate $\phi_{\mathrm{R}}$ a self-consistent solution of eq 2 is required, since $\phi_{\mathrm{R}}$ appears explicitly on the left side and implicitly on the right side through $\rho_{0}\left(\mathbf{r} ;\left[\phi_{\mathrm{R}}\right], \mu_{0}^{\prime}\right)$. Thus, a useful implementation of the MF idea requires a way to accurately determine the density response $\rho_{0}\left(\mathbf{r} ;\left[\phi_{\mathrm{R}}\right], \mu_{0}^{\prime}\right)$ induced by a given $\phi_{R}$.

B. Local Response to ERF. The classical VDW interface equation results when a second approximation, appropriate only for a slowly varying field, is used to estimate the density response $\rho_{0}\left(\mathbf{r} ;\left[\phi_{\mathrm{R}}\right], \mu_{0}\right)$. This hydrostatic approximation for the density takes account only of the local value of the field through a shift in the chemical potential. ${ }^{2,4,5}$ Thus $\rho_{0}\left(\mathbf{r}_{1} ;\left[\phi_{\mathrm{R}}\right]\right.$, $\mu_{0}^{\prime}$ ) is approximated for each $\mathbf{r}_{1}$ by $\rho_{0}^{\mathbf{r} 1}$, the local hydrostatic density response, which satisfies

$$
\mu_{0}\left(\rho_{0}^{\mathbf{r} 1}\right)=\mu_{0}^{\prime}-\phi_{\mathrm{R}}\left(\mathbf{r}_{1}\right)
$$

Hence the nonuniform density $\rho_{0}\left(\mathbf{r}_{1}\right)$ at each $\mathbf{r}_{1}$ is assumed to equal $\rho_{0}^{\mathbf{r} 1} \equiv \rho_{0}\left(\boldsymbol{r}_{1} ;[0], \mu_{0}^{\mathbf{r l}}\right)$, the density of the uniform reference fluid in zero field at the shifted chemical potential $\mu_{0}^{\mathrm{r} 1} \equiv \mu_{0}^{\prime}-$ $\phi_{\mathrm{R}}\left(\mathbf{r}_{1}\right)$, given by the right side of eq 5 . When $\phi_{\mathrm{R}}\left(\mathbf{r}_{1}\right)$ is a constant, this gives the exact result.

The superscript $\mathbf{r}_{1}$ in $\rho_{0}^{\mathbf{r} 1}$ is meant to remind us that $\rho_{0}^{\mathbf{r} 1}$, like $\rho_{0}^{\prime}$ or $\rho_{0}^{v}$, represents the density of the uniform reference fluid at a particular chemical potential $\mu_{0}^{\mathrm{r} 1}$, which from eq 5 depends parametrically on $\mathbf{r}_{1}$ through the local value of the ERF. Thus, when $\mathbf{r}_{1}$ is in the bulk liquid (vapor) phase then $\rho_{0}^{\mathbf{r} 1}$ reduces to $\rho_{0}^{\prime}\left(\rho_{0}^{v}\right)$. When $\phi_{\mathrm{R}}$ is very slowly varying, this "local field" approximation is quite accurate and in this special case is equivalent to the local density approximation made in the usual interpretation of the VDW theory. ${ }^{7,17}$ However, this approximation ignores the nonlocal excluded volume correlations that can be induced by a more rapidly varying $\phi_{\mathrm{R}}$. This represents a major limitation of the classical theory in more general applications and will be corrected in the MVDW theory.

C. Classical Interface Equation. The classical VDW interface equation follows immediately when $\rho_{0}\left(\mathbf{r}_{2}\right)$ is replaced by $\rho_{0}^{\mathbf{r} 2}$ in eq 2 , and the latter is substituted into eq 5 . This yields an integral equation for $\rho_{0}^{\mathbf{r} 1}$, which from eq 1 is supposed to equal the density in the full LJ fluid:

$$
\mu_{0}\left(\rho_{0}^{\mathbf{r} 1}\right)=\mu_{0}^{\prime}-\phi\left(\mathbf{r}_{1}\right)-\int \mathrm{d} \mathbf{r}_{2} \rho_{0}^{\mathbf{r}^{2}} u_{1}\left(r_{12}\right)-2 a \rho^{\prime}
$$

This can be exactly rewritten in a more standard form using $\mu(\rho)$ as defined in eq 4 :

$$
\mu\left(\rho_{0}^{\mathbf{r} 1}\right)=\mu^{\prime}-\phi\left(\mathbf{r}_{1}\right)-\int \mathrm{d} \mathbf{r}_{2}\left[\rho_{0}^{\mathbf{r} 2}-\rho_{0}^{\mathbf{r} 1}\right] u_{1}\left(r_{12}\right)
$$

Specializing to the case of the LV interface with planar symmetry and $\phi=0$ and expanding $\rho_{0}^{\mathbf{r} 2}$ to second order in a Taylor series about $\rho_{0}^{\mathbf{r} 1}$ (consistent with the assumption of a slowly varying interface) yields the classical VDW differential equation for the interface profile $\rho_{0}^{\mathrm{z}}$ :

$$
\mu\left(\rho_{0}^{\mathrm{z}}\right)-\mu^{\prime}=m \frac{d^{2} \rho_{0}^{\mathrm{z}}}{d z^{2}}
$$

where

$$
m \equiv-\frac{1}{6} \int \mathrm{d} \mathbf{r} r^{2} u_{1}(r)
$$


Equations 7 and 8 are completely equivalent to the VDW theory for the LV interface as it is usually presented. ${ }^{7}$ In our derivation the theory describes hydrostatic densities in the reference system, and $\mu(\rho)$ is also defined in terms of reference system quantities given on the right side of eq 4 . This provides a simple and consistent interpretation of the classical theory that avoids all of the conceptual problems associated with densities in the two phase region of the LJ fluid that arise in traditional approaches.

In this derivation we have obtained the VDW interface equation directly, without first approximating the free energy. We will later show how to determine the interface free energy in this approach. First we will discuss our new MVDW theory for the interface profile, which improves on both approximations made in the classical theory.

\section{MVDW Theory for the LV Interface Profile}

In the limit of a uniform system, eq 2 describes all effects of attractive interactions in terms of the constant parameter $a$ as in the van der Waals equation. While this very simple approximation captures much essential physics and gives a qualitative description of the uniform fluid thermodynamic properties, it certainly is not quantitatively accurate. In particular, when used to describe a slowly varying liquid-vapor interface, it will predict shifted (molecular field) values for the densities of the coexisting bulk liquid and vapor phases. The main problem with the classical theory in this case is not so much its description of the local density gradients, which are often small, but its predictions for the thermodynamic properties of the coexisting bulk phases. The first correction made in the MVDW theory is to modify eq 2 so that it agrees with a given equation of state for the uniform system while still giving reasonable results for nonuniform systems. ${ }^{3}$

A. Modified Molecular Field Approximation. To achieve quantitative agreement with known thermodynamic properties of the uniform LJ system we can replace the constant $a$ by a function $\alpha$ that depends (hopefully weakly, to the extent that the van der Waals theory is reasonably accurate) on temperature and density. ${ }^{3}$ Thus, instead of using the MF approximation for $\mu(\rho)$ as in eq 4 , we assume that $\mu(\rho)$ is known from an accurate bulk equation of state. In particular, we determine $\mu(\rho)$ from the 33-parameter equation of state for the LJ fluid given by Johnson et al. ${ }^{18}$ This provides a very good global description of the stable liquid and vapor phases in the LJ fluid and provides a smooth interpolation between the phases by using analytic fitting functions. Thus it naturally produces a modified "van der Waals loop" in the two-phase region and seems quite appropriate for our use here in improving the simplest MF description of the uniform fluid.

Now we relate this accurate $\mu(\rho)$ to the known $\mu_{0}(\rho)$ through a function $\alpha(\rho)$ defined so that

$$
\mu(\rho)=\mu_{0}(\rho)-2 \rho \alpha(\rho)
$$

Thus the exact chemical potentials in the uniform LJ and reference systems are related in the same way as is predicted by the simple MF approximation of eq 4, except that the constant $a$ is replaced by a (temperature and density dependent) function $\alpha(\rho)$ chosen so that eq 10 holds. We showed in ref 3 that the ratio $\alpha(\rho) / a$ is indeed of order unity and rather weakly dependent on density and temperature.

Because of the strictly local response in eq 5, these results for a constant field can also be used to determine exact results in the hydrostatic limit of a very slowly varying field. We want to modify eq 2 so that in the hydrostatic limit it will reproduce these exact values, while still giving reasonable MF results for more rapidly varying fields.

There is no unique way to do this, but the following simple prescription seems very natural and gives our final result, which we have called the modified molecular field (MMF) approximation for the $\mathrm{ERF}^{3}$ :

$$
\begin{array}{r}
\phi_{\mathrm{R}}\left(\boldsymbol{r}_{1}\right)-\phi\left(\boldsymbol{r}_{1}\right)=\frac{\alpha\left(\rho_{0}^{\mathbf{r} 1}\right)}{a} \int \mathrm{d} \boldsymbol{r}_{2} \rho_{0}\left(\boldsymbol{r}_{2} ;\left[\phi_{\mathrm{R}}\right], \mu_{0}^{\prime}\right) u_{1}\left(r_{12}\right)+ \\
2 \alpha\left(\rho^{\prime}\right) \rho^{\prime}
\end{array}
$$

Thus the molecular field integral in eq 2 is multiplied by a factor $\alpha\left(\rho_{0}^{\mathbf{r} 1}\right) / a$ of order unity that depends on $\mathbf{r}_{1}$ through the dependence of the hydrostatic density $\rho_{0}^{\mathbf{r} 1}$ on the local value of the field $\phi_{\mathrm{R}}\left(\mathbf{r}_{1}\right)$, and the constant $2 a \rho$ is replaced by the appropriate limiting value of the modified integral. The MVDW theory assumes that the ERF is given by eq 11 rather than eq 2 .

B. Nonlocal Response to the ERF. The second important correction made in the MVDW theory is to determine more accurately the full nonlocal response $\rho_{0}\left(\mathbf{r}_{1} ;\left[\phi_{\mathrm{R}}\right], \mu_{0}^{\prime}\right)$ to the ERF, thus correcting the local hydrostatic density $\rho_{0}^{\mathbf{r} 1}$ used in the classical theory. We introduced in ref 2 a simple and generally very accurate method for calculating the structure and thermodynamics of the reference fluid in the presence of a general external field, using linear response theory in a locally optimal way to calculate the nonlocal corrections to the hydrostatic density. For a very slowly varying field the theory gives the hydrostatic density and for a hard core field the theory naturally reduces to the Percus - Yevick approximation. ${ }^{19}$

The result is an integral equation for the density $\rho_{0}\left(\mathbf{r}_{1}\right)$, which we refer to as the hydrostatic linear response (HLR) equation:

$$
\rho_{0}\left(\mathbf{r}_{1}\right)=\rho_{0}^{\mathbf{r} 1}+\rho_{0}^{\mathbf{r} 1} \int d \mathbf{r}_{2} c_{0}\left(r_{12} ; \rho_{0}^{\mathbf{r} 1}\right)\left[\rho_{0}\left(\mathbf{r}_{2}\right)-\rho_{0}^{\mathbf{r} 1}\right]
$$

Here $c_{0}\left(r_{12} ; \rho_{0}^{\mathbf{r} 1}\right)$ is the direct correlation of the uniform reference fluid at the hydrostatic density $\rho_{0}^{\mathbf{r l}}$. This can be accurately approximated using known results for the uniform hard sphere fluid, as discussed in refs 2,4 . The $\mathbf{r}_{1}$ dependence of the linear response kernel $c_{0}$ through $\rho_{0}^{\mathbf{r} 1}$ is the most important new feature of the HLR equation. A discussion of the ideas leading to eq 12 and of its advantages over standard methods, along with numerical details of its solution, is given in refs 2 and 4.

C. Two-Step Method and the MVDW Theory. The MVDW theory for the LV interface arises from the selfconsistent solution of eqs 5, 11, and 12. A two-step iterative method proved sufficient in all cases tested. Given a starting guess for $\phi_{\mathrm{R}}\left(\mathbf{r}_{1}\right)$, one computes in the first step the local hydrostatic density response $\rho_{0}^{\mathbf{r l}}$ from eq 5 . Then in a second step nonlocal corrections leading to $\rho_{0}\left(\mathbf{r}_{1}\right)$ are determined from eq 12 , and this is used in eq 11 to give a new estimate for $\phi_{\mathrm{R}}\left(\mathbf{r}_{1}\right)$. This process is iterated to self-consistency, and accurate numerical results are readily obtained.

D. Simplified MVDW Interface Equation. A simplified version of the MVDW theory arises when one skips the second step and assumes that $\rho_{0}\left(\mathbf{r}_{1}\right)=\rho_{0}^{\mathbf{r} 1}$ as in the classical theory, while still using the accurate equation of state to determine $\mu(\rho)$ from eq 10. While the full MVDW theory is straightforward to implement, the nonlocal corrections for the LV interface are often small, and the use of the hydrostatic approximation allows for a more direct comparison with the classical theory. Using this approximation and eqs 11,5 , and 10 , we obtain an integral 
equation analogous to the classical eq 7. Expanding for simplicity to second order and assuming planar symmetry yields a generalization of the classical interface eq 8 :

$$
\mu\left(\rho_{0}^{\mathrm{z} 1}\right)-\mu^{\prime}=m_{\alpha}\left(\rho_{0}^{\mathrm{z} 1}\right) \mathrm{d}^{2} \rho_{0}^{\mathrm{z} 1} / \mathrm{d} z_{1}^{2},
$$

where

$$
m_{\alpha}(\rho) \equiv m \alpha(\rho) / a
$$

Following Rowlinson and $\mathrm{Widom}^{7}(\mathrm{RW})$, we can define

$$
\begin{aligned}
-W(\rho) & \equiv f(\rho)-\mu^{\prime} \rho+p^{\prime} \\
& =\rho\left[\mu(\rho)-\mu^{\prime}\right]-\left[p(\rho)-p^{\prime}\right]
\end{aligned}
$$

Here $f(\rho)$ is the Helmholtz free energy density from our analytic equation of state that corresponds to $\mu(\rho)$ given by eq 10 , where $\mu(\rho)=\mathrm{d} f(\rho) / \mathrm{d} \rho$ and the associated pressure $p(\rho)=-f(\rho)+$ $\rho \mu(\rho)$ from standard thermodynamics. We see that $W(\rho)$ vanishes in the coexisting bulk liquid and vapor phases and note that the left side of eq 13 is given by $-\mathrm{d} W\left(\rho_{0}^{\mathrm{z} 1}\right) / \mathrm{d} \rho_{0}^{\mathrm{z} 1}$. By interpreting the latter as a "force", eq 13 is analogous to Newton's law, with $\rho_{0}^{z 1}$ the "displacement", $z_{1}$ the "time", and $m_{\alpha}(\rho)$ a density (or "displacement") dependent "mass".

Note that eq 13 differs from the analogous equation that would arise in the classical theory from assuming a density dependent "mass" in the gradient correction to the free energy. As shown by RW in their eq 3.10, the latter would generate an additional square gradient term in the interface eq 13. It is difficult to see how such a term could arise naturally in our approach.

We report results here for the even simpler theory that arises when $m_{\alpha}(\rho)$ in eq 13 is replaced by its classical value $m$ given by eq 9 . The resulting simplified interface equation has the same form as the classical eq 8. However, it uses the accurate expression for $\mu(\rho)$ given by eq 10 , which ensures a proper thermodynamic description of the coexisting bulk phases, while (inconsistently) retaining the classical expression for $m$. This produces some changes in shape of the interface profile when compared to that predicted by the MVDW theory, but preserves many qualitative features such as the dependence of the interface width on the thermodynamic state and the range of the attractive interactions. One major virtue of this approximation is that a very simple expression for the profile $\rho_{0}^{\mathrm{z}}$ in terms of the inverse function $z\left(\rho_{0}\right)$ (with arbitrary origin) follows immediately from eq 13 by quadrature, as shown by RW:

$$
z\left(\rho_{0}\right)-z\left(\rho^{\mathrm{v}}\right)=\left(\frac{m}{2}\right)^{1 / 2} \int_{\rho_{v}}^{\rho_{0}} \mathrm{~d} \rho[-W(\rho)]^{-1 / 2}
$$

Results from this simplified approach and the full MVDW theory will be discussed in section $\mathrm{V}$ below.

E. Nonlocal Correlations and Capillary Waves. The use of the HLR equation in the MVDW theory allows one to take into account nonlocal correlations induced by the ERF. These arise mainly from the packing of the harshly repulsive molecular cores and become significant at high density when the ERF is rapidly varying. In principle, small amplitude excluded volume oscillations would be expected at high density from linear response theory far from any localized perturbation. ${ }^{20,21}$ The amplitude of these oscillations for a general liquid-vapor interface depends on the thermodynamic state and on the strength and range of the attractive interactions. These features control the steepness of the ERF $\phi_{R}(\mathbf{r})$, which mainly determines how significant these nonlocal corrections to the classical theory are in a given case.

For our study here of the LV interface in the LJ fluid, the attractive interactions are relatively slowly varying, and these corrections are numerically small in most cases. However, near the triple point the interface is very steep, and noticeable oscillations on the liquid side are predicted by the theory and, with much smaller amplitude, are also seen in the computer simulations. The classical local hydrostatic approximation precludes a description of any such oscillations and gives no indication of where it can break down. So these corrections are conceptually important even for "smooth" LV interfaces.

The difference in oscillation amplitude between theory and simulation arises because the MVDW theory describes a "static" interface in the reference fluid induced by the ERF. Any theory that takes account of attractive forces only through an ERF and uses reference system correlation functions to approximate structure in the LJ system cannot properly describe the physics leading to the long-wavelength capillary wave fluctuations that occur at a real LV interface. ${ }^{7,12,13}$ These induce characteristic long-ranged pair correlations in the interface region of a real fluid that are completely different from the corresponding pair correlations in the reference system, which remain short-ranged for any reasonable choice of $\phi_{\mathrm{R}}(\mathbf{r})$. In a sufficiently large system, the capillary wave fluctuations can wash out any excluded volume oscillations at the real LV interface, and indeed the entire interface profile $\rho(\mathbf{r})$ itself $^{12}$ !

However, the small system sizes studied in computer simulations or encountered in most experiments on interfaces in confined geometries cut off most effects of such capillary wave fluctuations. It is reasonable to interpret the MVDW theory as providing one way of defining an "intrinsic" profile unbroadened by capillary wave effects and to compare its predictions directly to the simulation or experimental data, taking account of the residual finite size capillary wave effects separately if necessary. ${ }^{13}$ This will be discussed further below.

Workers using other approaches such as density functional theory or integral equation methods sometimes argue that their theories describe correlations in the full nonuniform LJ system, and thus may include some or perhaps all of the effects of capillary wave fluctuations. ${ }^{22,23}$ However, such theories usually introduce approximations that relate correlation functions in the nonuniform system to interpolated or weighted correlation functions in the uniform LJ system. One must then deal with the ambiguities arising from unstable uniform densities in the two phase region. We believe most such arbitrary schemes implicitly introduce a mean field character to the theory through the use of uniform fluid correlation functions that do not contain any capillary wave effects. However the precise physical implications of such approximations are very difficult to assess. In our interpretation of MF theory, both the strengths and the limitations arising from the use of reference system correlation functions are clear from the outset.

\section{Results for Interface Structure}

A. MVDW Theory. Figure 1 shows the interface profiles for the LJ fluid for states at three different temperatures. The dots give results of recent molecular dynamics (MD) simulations of Mecke et al. ${ }^{8}$ They made a careful study of the important changes in the interface profile and surface tension that arise from setting the force from the LJ potential to zero beyond a certain cutoff distance $r_{\mathrm{c}}$. Since there are unbalanced attractive forces in the interface region, effects from different cutoffs are much more important than in uniform systems, where the 


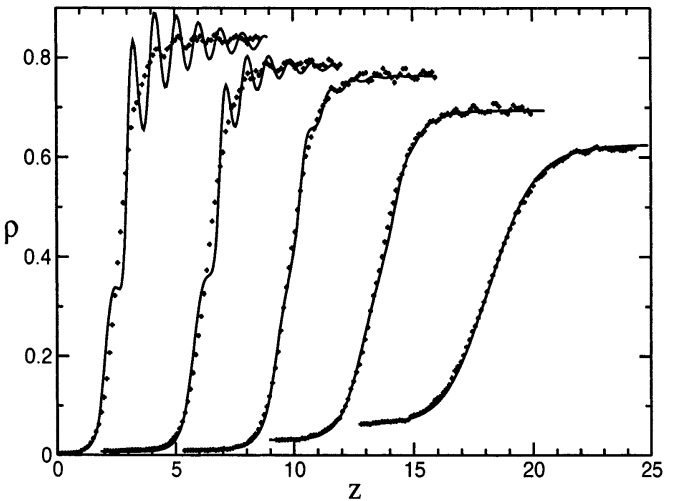

Figure 1. Density profiles of the LJ liquid-vapor interface for states with different temperatures and potential energy cutoffs. From left to right: $T=0.7$ and $r_{\mathrm{c}}=5.0 ; T=0.7$ and $r_{\mathrm{c}}=2.5 ; T=0.85$ and $r_{\mathrm{c}}=$ 5.0; $T=0.85$ and $r_{\mathrm{c}}=2.5 ; T=1.1$ and $r_{\mathrm{c}}=5.0$. Symbols are results of Mecke et al. ${ }^{8}$ Lines are predictions of the MVWD theory.

attractive forces essentially cancel. The first two curves on the left give profiles very near the triple point at a reduced temperature $T$ of 0.7 with $r_{\mathrm{c}}=5$ and 2.5 , respectively. There are notable changes in the coexistence densities $\rho^{v}$ and $\rho^{\prime}$ from the different cutoffs. The next two curves give results at $T=$ 0.85 with the same two cutoffs and the last curve on the right gives $T=1.1$ with $r_{\mathrm{c}}=5$.

The lines give results of the MVDW theory, using the appropriate cutoff and shifted LJ potential. The effects of the cutoff on the bulk thermodynamics can be taken into account in the general equation of state of Johnson et al. ${ }^{18}$ used in the MVDW theory, and the ERF properly describes the averaged effects of the unbalanced attractive forces in the interfacial region. Overall there is very good agreement between simulations and the theory, which captures all qualitative effects of changes in temperature and cutoff radius.

However, the theory predicts very noticeable density oscillations on the liquid side at the lowest temperature $T=0.7$. As discussed earlier, the amplitude of these oscillations is a sensitive function of temperature and cutoff, and already by $T=0.85$ their amplitude is greatly reduced. Such nonlocal excluded volume correlations are to be expected when the density is high and the ERF is sufficiently rapidly varying. In other contexts they play an important role in the physics of nonuniform fluids. ${ }^{5}$

Indeed we find that the theory provides an exceptionally accurate description of $\rho_{0}\left(\mathbf{r} ;\left[\phi_{\mathrm{R}}\right], \mu_{0}^{\prime}\right)$, the density of the reference system in the presence of the ERF $\phi_{R}$. This is illustrated in Figure 2, which compares the theory (solid lines) for $T=0.7$ and $T=0.75$ directly to results from grand canonical Monte Carlo simulations we carried out ${ }^{24}$ for the reference system density (symbols) in the presence of the self-consistently determined ERF (dotted lines). Note that while the field is smooth, it is sufficiently rapidly varying in this case to produce a density response with nonlocal excluded volume correlations. These are very well described theoretically by the HLR equation used in the MVDW theory and are completely missed by the local hydrostatic approximation (dashed lines) used in the classical theory.

The differences in oscillation amplitude seen in Figure 1 arise because the reference system cannot describe the capillary wave fluctuations seen at a real LV interface, as discussed above. Some residual effects are present even for the relatively small system sizes used in the computer simulations. We can take them into account in an approximate way by using the standard prescription for Gaussian capillary wave smearing of an "intrinsic" interface. Thus we convolute the "intrinsic" interface

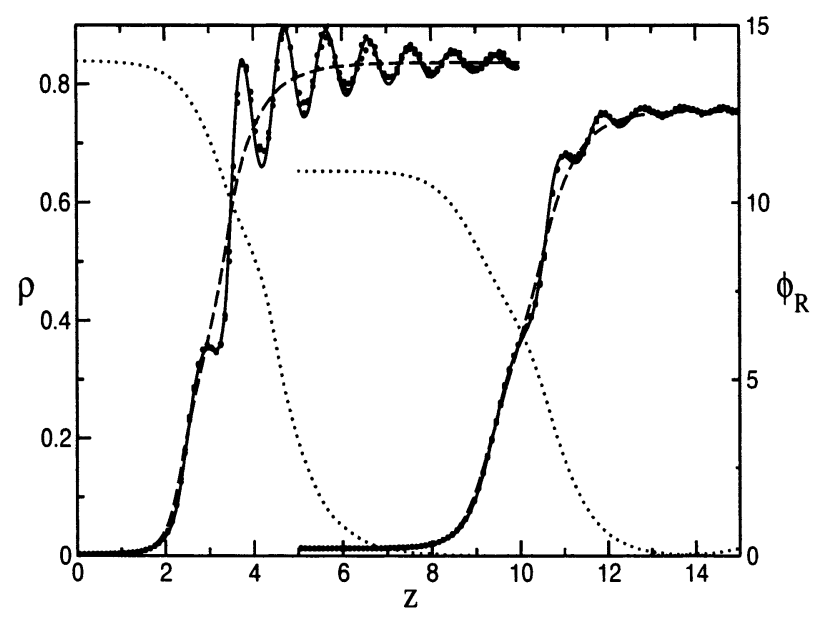

Figure 2. MC simulation of the reference system in the presence of the self-consistent field $\phi_{\mathrm{R}}$, given by the dotted lines (use the right vertical axis for fields). The full lines and symbols denote densities as in Figure 1. The dashed lines give the hydrostatic density defined by eq 5. The states are: (left) $T=0.7, r_{\mathrm{c}}=5.0$; (right) $T=0.75, r_{\mathrm{c}}=$ 2.5 .

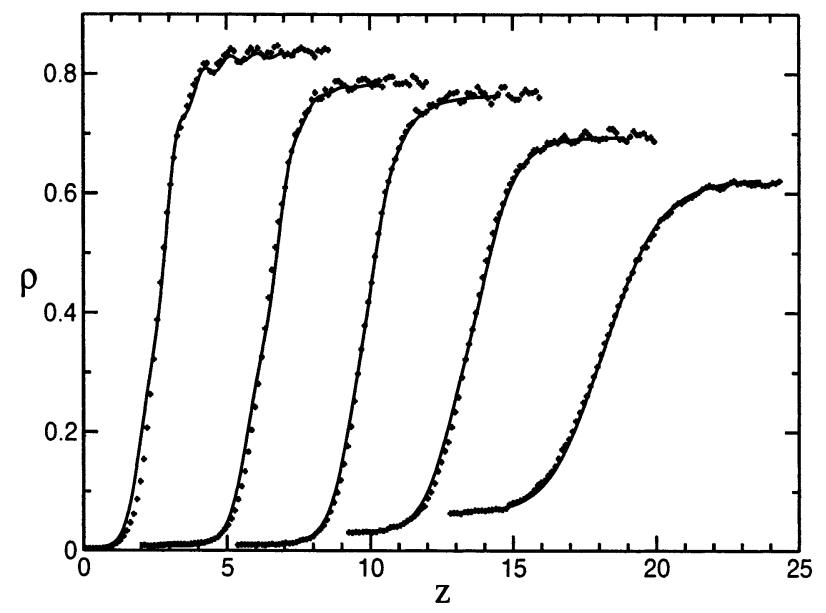

Figure 3. MVDW theory with capillary wave smoothing of profiles in Figure 1, with $L_{\mathrm{s}}$ equal to three times the interface width.

as given by the MVDW theory in Figure 1 with a Gaussian distribution of local interface positions $h$ :

$$
P(h)=\left(2 \pi s^{2}\right)^{-1 / 2} \exp \left(-h^{2} / 2 s^{2}\right)
$$

where the width $s$ of the Gaussian is given by

$$
s^{2}=\frac{k_{\mathrm{B}} T}{2 \pi \gamma} \ln \frac{L}{L_{\mathrm{s}}}
$$

Here $L$ is the lateral box size in the simulation and the $L_{\mathrm{S}}$ is the short distance (wavelength) cutoff, which is supposed to be proportional to the bulk correlation length or the interface width. ${ }^{7,12,13}$ In this case, the amount of smoothing depends mainly on the choice of $L_{\mathrm{s}}$, and the very reasonable choice of three times the interface width gives the data plotted in Figure 3 . The minimal choice of just the interface width gives too much smoothing when compared to the MD data.

We see that the finite size capillary wave fluctuations have very little effect on the smooth profiles at higher temperatures, but are quite effective in damping out some of the "intrinsic" oscillations predicted near the triple point. Our purpose here is not to advocate this particular and somewhat arbitrary prescription for smoothing the results of the MVDW theory, but to point 


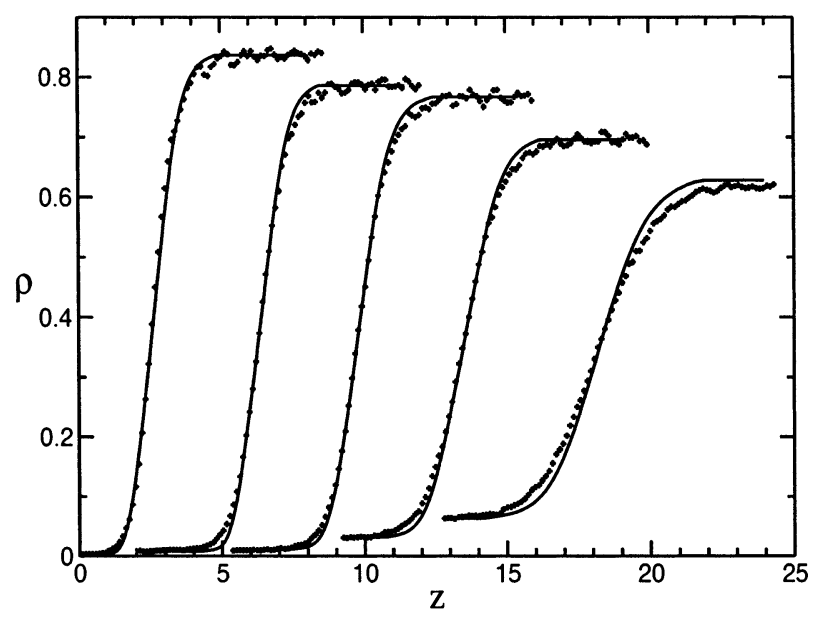

Figure 4. Simplified MVDW density profiles from eq 16. Same states as in Figure 1.

out that while excluded volume oscillations are accurately described by the MVDW theory, their influence on the LV interface profile is reduced by capillary wave effects not captured by the theory. In many other applications, e.g., structure near a hard wall or in a slit or pore, the capillary wave fluctuations are suppressed even further and the MVDW theory gives a good description of the excluded volume correlations such rapidly varying fields induce. ${ }^{5}$

B. Simplified MVDW Theory. Figure 4 gives the interface profiles predicted by the simplified MVDW theory from eq 16. The theory seems to give very good results, perhaps even better than those of the full MVDW theory in Figure 1! However, part of this agreement is a result of a fortuitous cancellation of errors. As shown in Figure 2, the hydrostatic approximation used in the simplified theory completely suppresses the excluded volume correlations that should really be present at $T=0.7$ in the reference system profile. As a result it seems to give better agreement with the simulation data for the LJ profile, where capillary wave effects, also not properly described by the simplified theory, wash out most effects of the oscillations. Note that the simplified theory gives least accurate results at $T=$ 1.1 where the interface is more slowly varying, and one would have expected the theory to be most accurate. As we will see later, results for the surface tension from the simplified theory are also less satisfactory. Nevertheless, the simplified theory captures well the qualitative effects of temperature and cutoff on the interface profile, and it is exceptionally easy to implement.

\section{Surface Tension}

A. Basic Formalism. In the MVDW theory, we first determine fluid structure. To calculate the interface free energy or surface tension $\gamma$ for the LV system, we proceed formally and imagine a special path where the density in the LJ fluid changes linearly ${ }^{25}$ from that of the uniform liquid with density $\rho^{\prime}$ to the final LV interface profile $\rho\left(\mathbf{r} ;[\phi=0], \mu^{\gamma}\right) \equiv \rho(\mathbf{r})$ as controlled by a coupling parameter $\lambda$ with $0 \leq \lambda \leq 1$ :

$$
\rho_{\lambda}(\mathbf{r})=\rho^{\prime}+\lambda\left[\rho(\mathbf{r})-\rho^{\prime}\right]
$$

Here $\rho_{\lambda}(\mathbf{r}) \equiv \rho\left(\mathbf{r} ;\left[\phi_{\lambda}\right], \mu^{\gamma}\right)$ where $\phi_{\lambda}(\mathbf{r})$ is the (generally nonzero) external field that formally produces the partially coupled profile $\rho_{\lambda}(\mathbf{r})$ defined by the right side of eq 19. Since $\rho_{\lambda}(\boldsymbol{r})=\delta \Omega_{\lambda} /$ $\delta \phi_{\lambda}(\boldsymbol{r})$, on integration the change in the Grand canonical free energy associated with this density change is exactly given by

$$
\Omega_{\lambda=1}-\Omega_{\lambda=0}=\int \mathrm{d} \mathbf{r} \int_{0}^{1} \mathrm{~d} \lambda \rho_{\lambda}(\mathbf{r}) \frac{\mathrm{d} \phi_{\lambda}(\mathbf{r})}{\mathrm{d} \lambda}
$$

Here $-\beta \Omega_{\lambda} \equiv \ln \Xi_{\lambda}$ where $\Xi_{\lambda}$ is the grand partition function for the system with field $\phi_{\lambda}$. For the LV interface we have $\phi_{\lambda=0}(\mathbf{r})=\phi_{\lambda=1}(\mathbf{r})=0$. Since $\Omega_{\lambda=0}=-p^{\prime} V$, with $p^{\prime}$ the coexistence pressure, which equals that in the vapor phase, the free energy difference on the left side is the desired interfacial free energy $\gamma$ and is independent of any choice of Gibbs dividing surface. ${ }^{7}$ Integrating by parts, we have our basic starting point:

$$
\gamma=-\int \mathrm{d} \mathbf{r} \int_{0}^{1} \mathrm{~d} \lambda \phi_{\lambda}(\mathbf{r}) \frac{\mathrm{d} \rho_{\lambda}(\mathbf{r})}{\mathrm{d} \lambda}
$$

or for the linear path:

$$
\gamma=-\int \mathrm{d} \mathbf{r}[\rho(\mathbf{r})-\rho] \int_{0}^{1} \mathrm{~d} \lambda \phi_{\lambda}(\mathbf{r})
$$

B. MVDW Theory for Surface Tension. The MF approximation used in the MVDW theory allows us to evaluate these expressions using reference system quantities. Thus we assume from eq 1 that $\rho_{\lambda}(\mathbf{r})=\rho_{0 \lambda}(\mathbf{r}) \equiv \rho_{0}\left(\mathbf{r} ;\left[\phi_{\mathrm{R} \lambda}\right], \mu_{0}^{\prime}\right)$, where $\phi_{\mathrm{R} \lambda}(\mathbf{r})$ is the field in the reference system producing the same profile, formally related to $\phi_{\lambda}(\mathbf{r})$ by the MMF eq 11 :

$$
\phi_{\mathrm{R} \lambda}\left(\boldsymbol{r}_{1}\right)=\phi_{\lambda}\left(\boldsymbol{r}_{\mathbf{1}}\right)+\frac{\alpha\left(\rho_{0 \lambda}^{\mathbf{r} 1}\right)}{a} \int \mathrm{d} \boldsymbol{r}_{2} \rho_{0 \lambda}\left(\boldsymbol{r}_{2}\right) u_{1}\left(r_{12}\right)+2 \alpha\left(\rho^{\prime}\right) \rho^{\prime}
$$

Using eq 5 and eq 10, we can exactly rewrite eq 23 in a convenient form for use in eqs 21 and 22:

$$
\phi_{\lambda}\left(\mathbf{r}_{1}\right)=\mu^{\prime}-\mu\left(\rho_{0 \lambda}^{\mathbf{r} 1}\right)-\frac{\alpha\left(\rho_{0 \lambda}^{\mathbf{r} 1}\right)}{a} \int \mathrm{d} \mathbf{r}_{2}\left[\rho_{0 \lambda}\left(\mathbf{r}_{2}\right)-\rho_{0 \lambda}^{\mathbf{r} 1}\right] u_{1}\left(r_{12}\right)
$$

In the MVDW theory $\mu(\rho)$ is determined from the accurate equation of state and is given by eq 10 , and all densities are calculated in the reference system.

To calculate $\phi_{\lambda}\left(\mathbf{r}_{1}\right)$ from eq 24 , we start with the final selfconsistent profile $\rho_{0}(\mathbf{r})$ and use eq 19 to define $\rho_{0 \lambda}(\mathbf{r})$. We then iterate the HLR eq 12 in an "inverse" way to find the hydrostatic density $\rho_{0 \lambda}^{\mathbf{r} 1}$ associated with a given $\rho_{0 \lambda}\left(\mathbf{r}_{1}\right)$. (Although we know that $\rho_{0 \lambda}(\mathbf{r})$ is linear in $\lambda$ from eq 19 , unless the density is slowly varying this does not imply that the same condition holds for $\rho_{0 \lambda}^{\mathbf{r}}$.) With this in hand, we use eq 24 to determine $\phi_{\lambda}\left(\mathbf{r}_{1}\right)$ for several intermediate values of $\lambda$. The surface tension is then calculated by carrying out the integration in eq 22 numerically.

C. Simplified Hydrostatic Approximations for the Surface Tension. If we ignore the difference between $\rho_{0 \lambda}\left(\mathbf{r}_{1}\right)$ and $\rho_{0 \lambda}^{\mathbf{r} 1}$ as in the classical theory and assume the latter varies linearly in $\lambda$, then we require only the final profile $\rho_{0}^{\mathrm{rl}}$ and can carry out most of the $\lambda$ integration in eq 21 analytically. We have already seen that this is a rather accurate approximation for the structure of the LV interface, and we use this approximation only under an integral in computing the free energy. As we will see, this greatly simplifies the calculation of the free energy and also allows us to make contact with classical results and the simplified MVDW theory.

Assuming that $\rho_{0 \lambda}\left(\mathbf{r}_{2}\right)=\rho_{0 \lambda}^{\mathbf{r} 2}$ in eq 24 we have

$$
\phi_{\lambda}\left(\mathbf{r}_{1}\right)=\mu^{\prime}-\mu\left(\rho_{0 \lambda}^{\mathbf{r} 1}\right)-\frac{\alpha\left(\rho_{0 \lambda}^{\mathbf{r} 1}\right)}{a} \int \mathrm{d} \mathbf{r}_{2}\left[\rho_{0 \lambda}^{\mathbf{r} 2}-\rho_{0 \lambda}^{\mathbf{r} 1}\right] u_{1}\left(r_{12}\right)
$$

and we will use this expression in eq 21 or 22 to determine the 
surface tension. For $\lambda=1$ we have $\phi_{\lambda=1}\left(\mathbf{r}_{1}\right)=0$, and eq 25 reduces to the generalized interface eq 13 on expanding $\rho_{0 \lambda}^{\mathbf{r} 2}$ to second order about $\rho_{0 \lambda}^{\mathbf{r} 1}$. The simplified MVDW interface equation discussed earlier, whose solution is given in eq 16 , follows on further approximating $\alpha(\rho)$ by $a$ in eq 25 or $m_{\alpha}$ by $m$ in eq 13.

Now let us carry out the $\lambda$ integration in eq 21 using eq 25 for $\phi_{\lambda}\left(\mathbf{r}_{1}\right)$. A clear discussion of most of the technical issues is given by RW. The first two terms on the right in eq 25 represent the local contribution to the interface free energy and can be integrated analytically:

$$
\begin{aligned}
I_{\text {local }} & \equiv-\int \mathrm{d} \mathbf{r} \int_{0}^{1} \mathrm{~d} \lambda \frac{\mathrm{d} \rho_{0 \lambda}^{\mathbf{r}}}{\mathrm{d} \lambda}\left[\mu^{\prime}-\mu\left(\rho_{0 \lambda}^{\mathbf{r}}\right)\right] \\
& =-\int \mathrm{d} \mathbf{r} \int_{\rho^{\prime}}^{\rho} \mathrm{d} \rho_{0 \lambda}^{\mathbf{r}} \frac{\mathrm{d} W\left(\rho_{0 \lambda}^{\mathbf{r}}\right)}{\mathrm{d} \rho_{0 \lambda}^{\mathbf{r}}} \\
& =-\int \mathrm{d} \mathbf{r} W\left(\rho_{0}^{\mathbf{r}}\right)
\end{aligned}
$$

where $W(\rho)$ is given in eq 15 .

The last term in eq 25 gives the nonlocal contribution to the free energy. Using eq 19 for $\rho_{0 \lambda}^{\mathbf{r}}$ we require only $\rho_{0}^{\mathbf{r}}$, and from eq 22 we find

$$
I_{\text {nonlocal }}=\int \mathrm{d} \mathbf{r}_{1}\left[\rho_{0}^{\mathbf{r} 1}-\rho^{\prime}\right] K\left(\mathbf{r}_{1}\right) \int \mathrm{d} \mathbf{r}_{2}\left[\rho_{0}^{\mathbf{r}^{2}}-\rho_{0}^{\mathbf{r} 1}\right] u_{1}\left(r_{12}\right)
$$

where

$$
K(\mathbf{r}) \equiv 2 \int_{0}^{1} \mathrm{~d} \lambda \lambda \alpha\left(\rho_{0 \lambda}^{\mathbf{r}}\right) / a
$$

Thus we have

$$
\gamma=I_{\text {local }}+I_{\text {nonlocal }}
$$

and both terms require only $\rho_{0}^{\mathbf{r}}$. In the simplified MVDW theory discussed earlier, we set $\alpha=a$ or $K(\mathbf{r})=1$ in eq 27 and expand $\rho_{0}^{\mathbf{r} 2}$ about $\rho_{0}^{\mathbf{r} 1}$ to second order. Using eq 13 with $m_{\alpha}=$ $m$, we see that these additional approximations imply that $I_{\text {nonlocal }}$ $=I_{\text {local }}$, as given in eq 26 . RW show in this case we can use the even simpler expression

$$
\gamma=\int_{\rho^{v}}^{\rho^{\prime}} \mathrm{d} \rho[-2 m W(\rho)]^{1 / 2}
$$

which does not require explicit knowledge of $\rho_{0}^{\mathbf{r}}$.

D. Results. Figure 5 gives the surface tension predicted by the MVDW theory (with no capillary wave smoothing of the profile), as well as that arising from the use of the hydrostatic approximation as in eqs 26 and 27, and the predictions of the simplified MVDW theory as in eq 30 . We see that the MVDW theory gives very good agreement with the simulation data. The simplified MVDW theory is much less accurate. Since all theories give essentially the same results for $I_{\text {local }}$, the problem with the simplified theory must arise from its treatment of nonlocal effects through the approximation $I_{\text {nonlocal }}=I_{\text {local }}$, which we see becomes increasingly inaccurate at lower temperatures. Equation 27 provides a more accurate but still simple alternative to use of the full MVDW theory.

\section{Conclusions}

The MVDW theory provides a simple and physically motivated approach that can describe the structure and thermo-

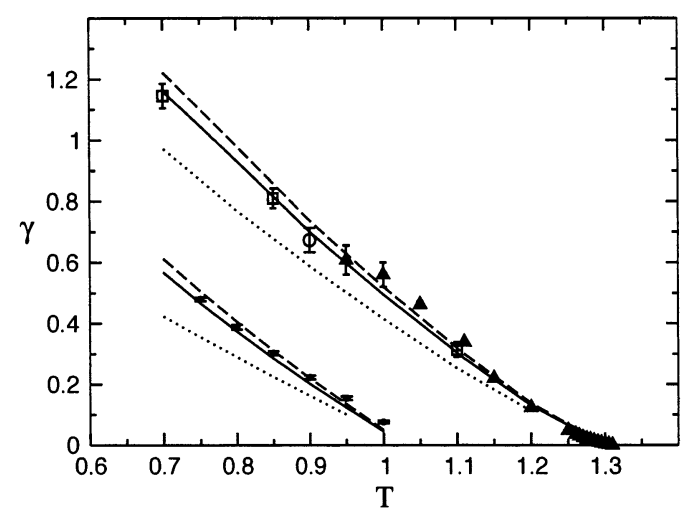

Figure 5. Surface tension $\gamma$ of the LJ liquid-vapor interface. The two sets correspond to the full LJ interaction potential (upper) and the cut-and-shifted LJ interaction with $r_{\mathrm{c}}=2.5$ (lower). Full lines are MWVD predictions. Dashed lines use the hydrostatic density to compute $I_{\text {nonlocal }}$ from eq 27. Dotted lines are results of the simplified MVDW theory from eq 30. Results of simulations are from Mecke et al. ${ }^{8}$ (squares), Holcomb et al..$^{9}$ (empty circle), Haye et al. ${ }^{10}$ (filled circles), and Potoff et al. ${ }^{11}$ (filled triangles).

dynamics of a fluid in a general external field. It optimally combines two standard approximations, a molecular field treatment of attractive interactions, modified to give accurate thermodynamic data for the uniform fluid, along with a linear response treatment of correlations in the reference fluid. The present application of the theory to the LV interface permits a new interpretation of the classical VDW theory that removes some ambiguities in standard treatments and shows how key features of the classical theory can be improved in a natural way. The accuracy of the MVDW theory in this application provided additional support for the physical ideas behind the theory and for its quantitative utility.

Acknowledgment. It is a pleasure to dedicate this paper to John Tully on the happy occasion of his 60th birthday. This work is supported by the National Science Foundation through Grant CHE-0111104. We are grateful to Yng-Gwei Chen and Jim Henderson for helpful discussions, and thank J. Winkelmann for sending us his computer simulation data.

\section{References and Notes} 4400

(1) Weeks, J. D.; Katsov, K.; Vollmayr, K. Phys. Rev. Lett. 1998, 81,

(2) Katsov, K.; Weeks, J. D. Phys. Rev. Lett. 2001, 86, 440.

(3) Katsov, K.; Weeks, J. D. J. Phys. Chem. B 2001, 105, 6738.

(4) Vollmayr-Lee, K.; Katsov, K.; Weeks, J. D. J. Chem. Phys. 2001, $114,416$.

(5) Weeks, J. D. Annu. Rev. Phys. Chem. 2002, 53, 533

(6) Rowlinson, J. J. Stat. Phys. 1979, 20, 197 gives an accessible English translation of the original VDW article, first published in 1893, along with some interesting commentary.

(7) Rowlinson, J. S.; Widom, B. Molecular Theory of Capillarity; Clarendon Press: Oxford, 1989.

(8) Mecke, M.; Winkelmann, J.; Fischer, J. J. Chem. Phys. 1997, 107, 9264.

(9) Holcombe, C. D.; Clancy, P.; Zollweg, J. A. Mol. Phys. 1993, 78, 437.

(10) Haye, M. J.; Bruin, C. J. Chem. Phys. 1994, 100, 556

(11) Potoff, J. J.; Panagiotopoulos, A. Z. J. Chem. Phys. 2000, 112, 6411.

(12) Buff, F. P.; Lovett, R. A.; Stillinger, F. H. Phys. Rev. Lett. 1965 15,621 .

(13) Weeks, J. D. J. Chem. Phys. 1977, 67, 3106.

(14) Weeks, J. D.; Selinger, R. L. B.; Broughton, J. Q. Phys. Rev. Lett. 1995, 75, 2694.

(15) Sullivan, D.; Stell, G. J. Chem. Phys. 1978, 69, 5450. 
(16) Weeks, J. D.; Vollmayr, K.; Katsov, K. Physica A 1997, 244, 461.

(17) Evans, R. In Fundamentals of Inhomogeneous Fluids; Henderson, D., Ed.; Dekker: New York, 1992; p 85.

(18) Johnson, J. K.; Zollweg, J. A.; Gubbins, K. E. Mol. Phys. 1993, $78,591$.

(19) Hansen, J. P.; McDonald, I. R. Theory of Simple Liquids; Academic Press: London, 1986.

(20) Evans, R.; Leote de Carvalho, R. J. F.; Henderson, J. R.; Hoyle, D. C. J. Chem. Phys. 1994, 100, 591.

(21) Evans, R.; Henderson, J. R.; Hoyle, D. C.; Parry, A. O.; Sabeur, Z. A. Mol. Phys. 1993, 80, 755.
(22) Iatsevitch, S.; Forstmann, F. J. Chem. Phys. 1997, 107, 6925.

(23) Iatsevitch, S.; Forstmann, F. Mol. Phys. 2000, 98, 1309.

(24) We carried out a standard grand canonical MC simulation. There are three types of moves: insertion of a particle, removal of a particle, and a Canonical local movement of a particle. The ratio of the moves was 1:1: 20 , which we did not try to optimize. Both a hard sphere fluid of appropriate diameter in the field and the reference LJ fluid in the same field were studied. Both simulations give essentially indistinguishable results. The hard sphere simulation is considerably faster and the figure has those results.

(25) Other density paths can be used, and in some cases may be preferable. See Y.-G. Chen and J. D. Weeks, to be published. 